\title{
The development of a real-time PCR assay for the rapid identification of Candida auris
}

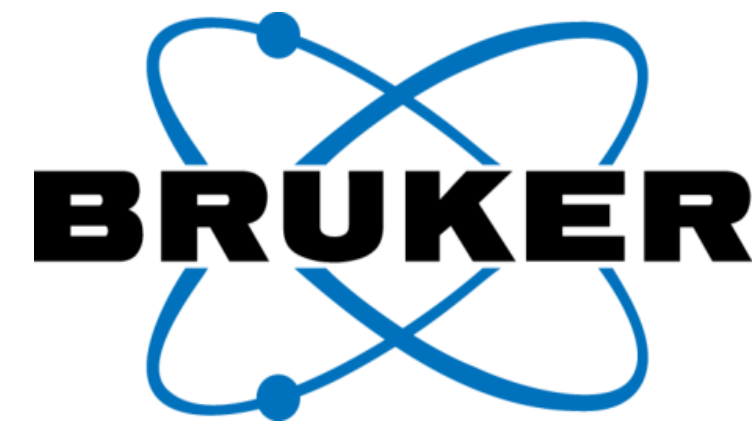

Selina Henriquez, Kate Dempsey, Gillian Miller \& Julie Green*

Bruker Microbiology, Glasgow, UK julie.green@bruker.com

\section{Introduction}

Candida auris is an emerging agent of invasive fungal infection, which is often multi-drug resistant. It has been responsible for outbreaks in healthcare settings worldwide. These outbreaks are difficult to control, even with enhanced measures in place and unlike other Candida species, C. auris is highly transmissible from patient to patient. Laboratory identification of Candida auris can be difficult because although culture methods can be quite sensitive, it can still take 24 hours to achieve growth and identification from culture can be problematic. While Bruker's MALDI Biotyper ${ }^{\circledR}$ provides unambiguous identification of $C$. auris, other techniques can misidentify Candida auris as Candida haemulonii. Real-time PCR methods for the detection of $C$. auris could provide quick results for environmental screening, colonisation or infection, and help to control spread in the clinical setting.

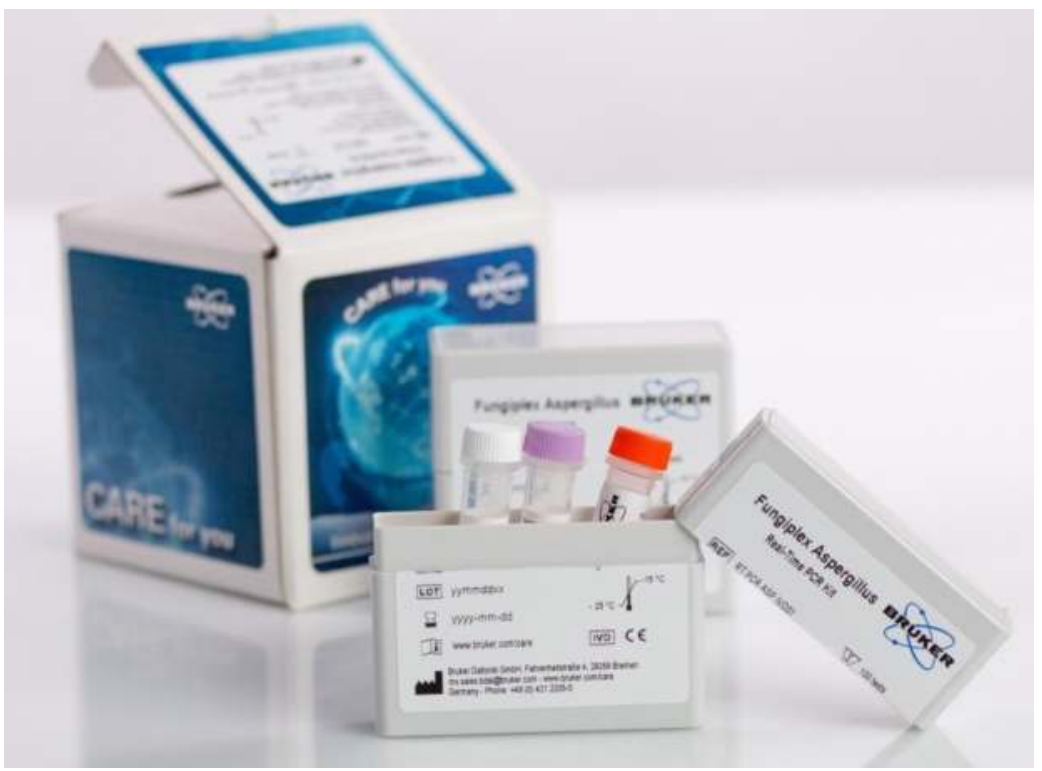

Fig 1: Example of a Fungiplex ${ }^{\circledR}$ Real-Time PCR Kit

A specific Candida auris PCR within Bruker's existing Fungiple ${ }^{\circledR}$ product portfolio would allow highly sensitive, highly specific detection without culture. An example Fungiplex Kit is shown in Figure 1.

\section{Methods}

For Candida auris detection the primers and probes were designed to target the Mating Factor $\alpha 1$ gene (MFa1) of $C$. auris which was found to be highly specific ${ }^{1}$.

The analytical performance and Limit of detection (LOD) of the Fungiplex Candida auris assay was determined by testing plasmid DNA between $20-2 \times 10^{6}$ input copies (ipc) as well as gDNA extracted from simulated samples in serum, plasma or directly from swab. For the preparation of simulated samples cultured material grown for $C$. auris over 2 days was diluted before adding to the samples. The simulated swab samples were extracted via two methods; (1) direct extraction using a Qiagen EZ1, and (2) boiled lysate ${ }^{2}$.

Sensitivity analysis was performed against 28 different Candida auris strains in reps of 3 . Specificity was assessed in a cross reactivity study using high concentration DNA extracted from cultured organism.
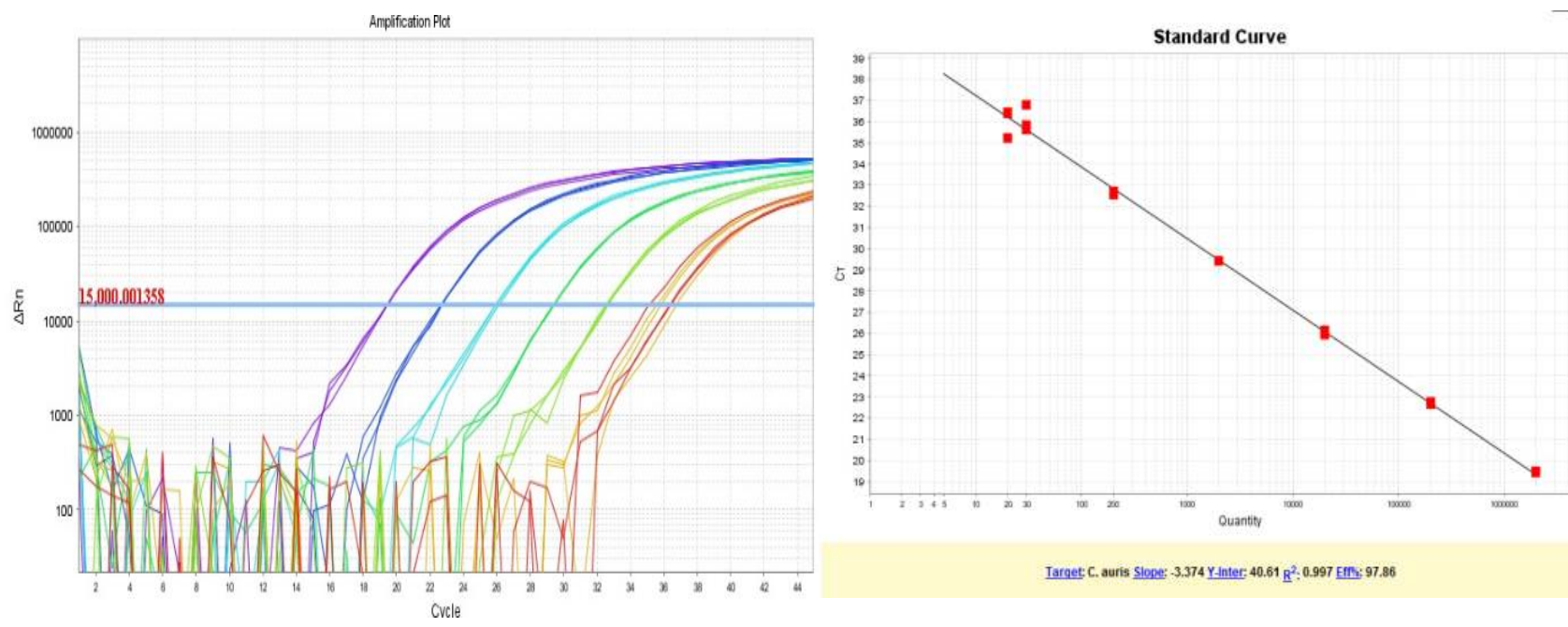

Fig 2: Fungiplex Candida auris Real-Time PCR dynamic range of $20-2 \times 10^{6} \mathrm{ipc}$ with plasmid DNA on the ABI7500
Results of the analytical performance are shown in Figure 2, where detection of Candida auris is reproducibly achieved down to 20 input copies. When further testing the sensitivity of the assay, the detectable concentrations using extracted gDNA are shown to be between $10-28$ input copies, depending on the extraction method used (Table 1).

Table 1: Number of $C$. auris copies of gDNA calculated via Standard Curve Method from swabs (Transwab) simulated samples extracted either via boiled lysate or commercial extraction (EZ1 extraction system)

\begin{tabular}{|c|c|c|c|c|c|}
\hline \multicolumn{3}{|c|}{ Boiled lysate } & \multicolumn{3}{c|}{ Commercial extraction } \\
\hline Sample & $\begin{array}{c}\text { Number } \\
\text { of Copies }\end{array}$ & $\begin{array}{c}\text { Detected } \\
\text { in reps of 3 }\end{array}$ & Sample & $\begin{array}{c}\text { Number } \\
\text { of Copies }\end{array}$ & $\begin{array}{c}\text { Detected } \\
\text { in reps of 3 }\end{array}$ \\
\hline $1: 10$ & 86,391 & $\checkmark$ & $1: 10$ & $1,179,515$ & $\checkmark$ \\
\hline $1: 100$ & 72,177 & $\checkmark$ & $1: 100$ & 399,962 & $\checkmark$ \\
\hline $1: 1000$ & 42,810 & $\checkmark$ & $1: 1000$ & 5,885 & $\checkmark$ \\
\hline $1: 10000$ & 1,992 & $\checkmark$ & $1: 10000$ & 542 & $\checkmark$ \\
\hline $1: 100000$ & 28 & $\checkmark$ & $1: 10000$ & 10 & $\checkmark$ \\
\hline
\end{tabular}

Of the 28 Candida auris strains tested all were detected in 3 replicate samples resulting in an analytical sensitivity of $100 \%$ (data not shown).

No cross reactivity was observed, resulting in an analytical specificity of $100 \%$. The species tested for cross reactivity are shown in Table 2.

Table 2: Results of the cross reactivity study for the Fungiplex Candida auris assay using 29 strains across different species

\begin{tabular}{|l|c|}
\hline Species tested & $\begin{array}{c}\text { Positivity / shown to } \\
\text { cross react }\end{array}$ \\
\hline Candida haemulonii (8 strains) & $\mathbf{x}$ \\
Candida haemulonis (2 strains) & $\mathbf{x}$ \\
Candida duobushaemulonii (6 strains) & $\mathbf{x}$ \\
Candida dubliniensis & $\mathbf{x}$ \\
Candida lipolytica & $\mathbf{x}$ \\
Candida lusitanie & $\mathbf{x}$ \\
Candida albicans & $\mathbf{x}$ \\
Candida glabrata & $\mathbf{x}$ \\
Candida krusei & $\mathbf{x}$ \\
Candida sake & $\mathbf{x}$ \\
Candida parapsilosis & $\mathbf{x}$ \\
Kodameae ohmeri & $\mathbf{x}$ \\
Rhodotorula glutinis & $\mathbf{x}$ \\
Saccharomyces cerevisiae & $\mathbf{x}$ \\
Aspergillus fumigatus & $\mathbf{x}$ \\
Extracted human serum & $\mathbf{x}$ \\
\hline
\end{tabular}

\section{Conclusions}

- Fungiplex Candida auris reliably detects Candida auris and is proven across multiple strains from a wide geographic spread

- Analytical sensitivity and specificity is $\mathbf{1 0 0} \%$ for Candida auris

- The Fungiplex Candida auris RUO Real-Time PCR assay can be used for environmental screening as well as detecting colonisation in clinical settings

\section{References}

(1) S. L. Panwar, M. Legrand, D. Dignard, M. Whiteway, and P. T. Magee; Eukaryotic Cell, Dec, 2003, 1350-1360

(2) L. Leach, Y. Zhu, S. Chaturvedi; Journal of Clinical Microbiology. 2018;56(2):e01223-17

\section{Acknowledgements}

Strains for this analysis were kindly supplied by -

- Dr. Jacques Meis (Canisius Wilhelmina Hospital, Nijmegen)

Dr. Anuradha Chowdhary (Vallabhbhai Patel Chest Institute, Delhi)

- Centre for Disease Control and Prevention (CDC challenge panel) 